\title{
LA NOCIÓN UNIVERSAL DEL DERECHO Y LOS \\ CONCEPTOS JURIDICOS FUNDAMENTALES
}

1. En este artículo me propongo examinar dos cuestiones: a) ¿Cuándo un concepto jurídico debe considerarse como fundamental? b) ¿Qué conceptos tienen el carácter de fundamentales en la órbita del derecho?

Los problemas a que dan expresión las anteriores preguntas han sido largamente discutidos en la literatura jurídica $y$, con especial amplitud $y$ hondura, en la germánica. Si los replanteamos, no es porque juzguemos que los criterios ofrecidos por la doctrina nos parezcan erróneos, sino porque las conclusiones a que los autores han llegado al aplicarlos están condicionadas por la noción del derecho que sirve de base a cada jurista. El método que emplean es casi siempre correcto, pero los resultados dependen -en su valor científico- de la definición de lo jurídico en que cada teoría descansa. Los autores que han calado más hondo en estos tópicos son, a no dudarlo, Austin, Somló y Stammler, y a ellos se debe la formulación de los criterios que permiten resolver los dos problemas; pero la noción del derecho que utilizan refleja sus limitaciones en los resultados que cada uno logra. Quien no acepte el punto de partida no puede admitir las conclusiones, ya que éstas encuéntranse condicionadas por aquél. Es posible seguir el método de que se valen los ilustres autores de Lectures on Jurisprudence, Juristische Grundlehre y Theorie der Rechtswissenschaft, ${ }^{1}$ y obtener, mediante el empleo de recursos metodológicos idénticos, resultados completamente diversos. Parece, pues, oportuno, examinar nuevamente los interrogantes que encabezan este artículo, pero a la luz de un diferente concepto del derecho. Al hacerlo, tenemos conciencia de que el valor de nuestro ensayo - si alguno tiene- dependerá, en forma casi exclusiva, del que pueda reclamar para sí la noción que habrá de servirnos de base. Como no sería posible -dentro de los estrechos límites de un artículo- tratar con la amplitud deseable esa cuestión, nos vemos obligados a remitir al lector a otros trabajos nuestros, en que el problema de la definición de lo jurídico ha sido examinado con todo el detenimiento que su importancia reclama. ${ }^{2}$

1 Cf. John Austin, The Uses of the Study of Jurisprudence, apéndice de The Province of Jurisprudence determined, The Noonday Press, New York, 1954; Félix Somlo, Juristische Grundlehre, Felix Meiner Verlag, Leipzig, Zweite Auflage, 1927; R. Stammler, Theorie der Rechtswissenschaft, Buchhandlung des Waisenhauses, Halle A. d. S., Zweite Auflage, 1923.

2 Eduardo García Máynez, El problema de la definición del Derecho, Stylo, México, 1948; Lógica del Juicio Jurídico, Fondo de Cultura Económica, México, 1955; Introducción al Estudio del Derecho, Editorial Porrúa Hermanos, México, $7^{\text {a }}$ ed., 1956. 
2. ¿Qué es lo que determina el carácter fundamental de un concepto jurídico? En opinión de Austin, la diferencia entre conceptos jurídicos fundamentales y no fundamentales deriva de la índole necesaria de los primeros. En el opúsculo titulado The Uses of the Study of Jurisprudence, ${ }^{3}$ después de sostener que el tema de la Jurisprudencia, en cualquiera de sus ramas, es el derecho positivo, declara que "si bien todo sistema jurídico tiene sus diferencias características y específicas, hay principios, conceptos y distinciones comunes a varios sistemas", ${ }^{4}$ y que esos principios, distinciones y conceptos forman el objeto de estudio de la Jurisprudencia General, que Austin distingue de la particular o nacional de cada país, lo mismo que de la política legislativa. ${ }^{5}$

Seguidamente enseña que de los principios, conceptos y distinciones a que está referida la General Jurisprudence, algunos pueden ser considerados como necesarios. Pues - añade- "no podemos pensar de modo coherente un sistema jurídico, sin ver en tales conceptos, distinciones y principios los elementos imprescindibles de tal sistema". Como ejemplos de conceptos fundamentales cita los de 'deber jurídico', 'derecho subjetivo', libertad', 'entuerto', 'castigo', etc.; entre las distinciones necesarias menciona la que existe entre derecho escrito y no escrito; la que media entre derechos subjetivos relativos y absolutos; la división de los absolutos en derecho de propiedad y desmembramientos de éste; la de las obligaciones en 'ex contractu' y 'quasi ex contractu'; la de los delitos en privados y públicos, etc. Tienen carácter básico, por último, ciertos principios implícitos en aquellos conceptos y distinciones. El jurista -escribe Austin- debe descubrirlos, a fin de desprender de ellos las consecuencias que encierran. ${ }^{7}$

Los principios, conceptos y distinciones cuyo estudio incumbe a la Jurisprudencia General no son todos necesarios. Cabe, en efecto, pensar de modo coherente un sistema de derecho, sin atribuir a todos ellos el mismo rango. Algunos son arbitrarios, por grandes que sean su generalidad o conveniencia práctica. Empero, es inútil tratar de expresarlos científicamente, si no se tiene un conocimiento preciso de los que sí son fundamentales. Nada impediría concebir, por ejemplo, un ordenamiento legal al que fuese ajena la división de los derechos privados en reales y personales; pero sería imposible pensar ninguno en que no existiesen derechos subjetivos, deberes jurídicos, sujetos de derecho, sanciones, etc. ${ }^{8}$

El criterio propuesto por Austin para diferenciar los conceptos, divisiones y principios fundamentales de los no fundamentales consiste, pues, en inqui-

3 The Uses of the Study of Jurisprudence, edición citada en la nota 1.

4 Austin, op. cit., pág. 365.

- Austin, op. cit., pág. 365 .

- Austin, op. cit., pág. 367.

7 Austin, op. cit., pág. 367.

8 Austin, op. cit., pág. 369. 
rir si tales conceptos, divisiones y principios en todo caso existen. En otras palabras: pertenecerán al primer grupo aquellos en cuya ausencia no podamos pensar un sistema de derecho; integrarán el segundo los que históricamente pueden existir o no existir.

Como muy bien lo ha advertido Somlo, échase de menos, en la tesis de Austin, un análisis profundo del sentido de la expresión 'conceptos necesarios', lo cual explica, por otra parte, el error en que el famoso autor incurre al incluir dentro de ese grupo algunos que no poseen el carácter que Austin les atribuye, como, por ejemplo, la división de las obligaciones en ex contractu, ex delictu y quasi ex contractu, o la contraposición de los conceptos de posesión y propiedad. ${ }^{2}$ Lo que en buena parte ha originado el error es — según el propio Somló - el distingo que el jurista británico introduce al hablar de 'conceptos necesarios de cualquier orden jurídico' y 'conceptos necesarios de los ordenamientos jurídicos evolucionados. ${ }^{10}$ De este modo mezcla ciertos contenidos generales con los elementos necesarios de orden formal de las nociones que maneja. No debe, empero, desconocerse que el autor de las célebres Lectures on Jurisprudence fue el primero en descubrir una de las características de los conceptos básicos: su índole necesaria, que los distingue de los que sólo valen dentro de ciertos sistemas, y a los que, por ello mismo, puede dárseles el calificativo de contingentes.

Falta, en cambio, en la doctrina austiniana, la distinción entre elementos materiales y formales de los principios y conceptos jurídicos, lo mismo que una idea precisa acerca del método que puede llevarnos al descubrimiento de los de carácter necesario. Estos perfeccionamientos no se encuentran en los enseñanzas de los discípulos del jurista inglés, que no se interesaron en profundizar la teoría de los conceptos fundamentales, sino en las obras de Rodolfo Stammler y Felix Somló.

3. Aun cuando admitamos que los conceptos jurídicos básicos se distinguen de todos los demás en que no pueden ser ignorados por ningún ordenamiento positivo, el criterio propuesto por Austin no basta para conocer la esencia de tales conceptos, ni permite determinar -a priori- cuáles ostentan ese rango. No se trata, en efecto, de establecer empíricamente qué nociones existen de modo indefectible, sino de saber cuáles no pueden ser contingentes, por tratarse de verdaderas categorías del pensamiento jurídico, esto es, de conceptos que condicionan el conocimiento del derecho. Hay que inquirir, en otras palabras, por qué tienen ese carácter de necesidad que Austin les atribuye, y de qué manera puede hacerse un inventario de los mismos.

La afirmación de que se trata de elementos formales del conocimiento normativo tampoco responde a estas preguntas, porque si bien es cierto que

9 Felix Soml6, op. cit., Einleitung, 11, pág. 33.

10 Somlo, op. cit., pág. 34. 
los conceptos básicos están referidos a formas de objetos jurídicos, no debe olvidarse que toda noción genérica, en el campo del derecho, refiérese también a formas, sin merecer por ello el calificativo de fundamental.

El concepto 'derecho de crédito', por ejemplo, está referido a todos los miembros de la clase designada por él, y los atributos que determinan la inclusión de un derecho subjetivo en dicha clase son siempre de orden formal. El contenido de cada uno de esos derechos tiene, en cambio, frente a los mencionados atributos, carácter material, y es el elemento variable de tales facultades. A pesar de ello, el concepto que las abarca no puede incluirse entre los fundamentales, ni conserva su carácter formal frente a la noción 'derecho subjetivo', que sí pertenece, como luego veremos, a la supradicha categoría.

Además de la relatividad - tantas veces señalada- de las expresiones forma y contenido, hay que tomar en cuenta que estos términos suelen ser empleados en sentidos muy diversos. Tal diversidad puede obedecer, por ejemplo, a la naturaleza de los objetos a que esas nociones se aplican. Háblase, verbigracia, de la 'forma' de una proposición o de un silogismo jurídicos, pero también de la de un deber jurídico o un derecho subjetivo. Es obvio que el vocablo no tiene el mismo alcance en las dos parejas de casos, por la diferente naturaleza de los correspondientes objetos. Cuando aludimos a la 'forma' de un juicio, de un concepto o de un razonamiento (jurídicos o de otra especie), tratamos de conocer la estructura ${ }^{11}$ de esas significaciones y, por tanto, el elemento invariable de ciertos objetos de orden lógico; cuando distinguimos los elementos materiales y formales de un deber o de un derecho, en cambio, no hacemos alusión a objetos lógicos, sino a los correlatos objetivos de las nociones 'deber jurídico' y 'derecho subjetivo'. Examinemos con mayor detenimiento este punto.

4. De 'formas' puede hablarse - y se ha hablado desde Aristóteles- en relación con objetos lógicos, es decir, relativamente a conceptos, juicios y raciocinios. A las de esta clase puede aplicárseles -en razón de su naturalezala denominación de discursivas, ya que solemos expresarlas por medio del lenguaje. Cabe inquirir cuál es la forma de un conoepto, cuál la de un juicio o la de un raciocinio $y$, en cualquiera de estos casos, se hace abstracción del contenido del concepto, del juicio o del raciocinio.

En su Geschichte der Logik, ${ }^{12}$ Heinrich Scholz ha precisado con admirable claridad las nociones de 'forma lógica' y 'forma lógica perfecta', implícitas en el Organon aristotélico y, por ende, el sentido en que -relativamente a objetos lógicos- cabe hablar de elementos materiales y formales.

11 Sobre el concepto de estructura véase la obra de S. Langer, An Introduction to Symbolic Logic, Dover Publications, New York, Second Edition, 1953; Cap. I, 3. $\$ 1$.

12 Heinrich Scholz, Geschichte der Logik, Junker und Dünnhaupt Verlag, Berlin, 1931; 
A fin de aclarar aquellos términos, presuponemos, con Aristóteles, "que estamos en condiciones de dividir los elementos integrantes de cualquier enunciado -entendiendo por tal, con el mismo filósofo, la expresión de la que puede afirmarse que es verdadera o falsa - en dos clases, de suerte que a la primera pertenezcan los elementos que juzgamos fijos o invariables, y a la segunda los que deseamos considerar como variables. A los últimos los designamos, también con Aristóteles, por medio de letras, e interpretamos las letras como signos de variables o, más brevemente, como variables, esto es, como signos para lugares vacíos en los que puede introducirse algo, de cuya naturaleza hacemos abstracción en un principio. Podemos, pues, decir esto: por 'forma', en general, entendemos una expresión en que aparece, al menos, una variable, de modo que al sustituir esa variable por algo o, para expresarlo de manera más precisa, al dar un contenido adecuado al lugar que ocupa, la correspondiente expresión se convierte en un enunciado verdadero o falso" ${ }^{13}$

'Forma perfecta' es la expresión que puede obtenerse de un enunciado "cuando sustituimos por signos idóneos todos los elementos del mismo enunciado que pueden considerarse como variables". ${ }^{14} \mathrm{El}$ ejemplo más sencillo de 'forma lógica perfecta', en la simbólica elemental del Estagirita, es la expre-

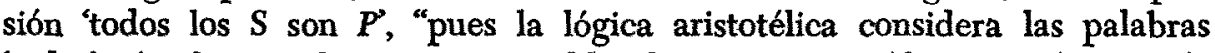
'todos' y 'son' como elementos invariables, lo que hace que la expresión cumpla las condiciones de una forma perfecta". Las de esta clase son las únicas de que se ocupa aquella lógica. Esto no quiere decir que convierta en objeto de estudio cualquiera de esas formas, sino sólo las que pueden quedar sometidas a ciertas reglas, llamadas 'de inferencia'. Una regla de esta naturaleza encierra siempre, para Aristóteles, tres formas perfectas $F_{1}, F_{2}, F_{3}$, de suerte que, si $F_{1}$ y $F_{2}$ pueden convertirse en proposiciones verdaderas, de su verdad cabe inferir la de la proposición que corresponde a $F_{3}$. En resumen: "la lógica aristotélica $o$, dicho con más rigor, la lógica a que Aristóteles dio un fundamento, es formal en la medida en que exclusivamente se ocupa con formas perfectas, que escoge de manera de poder formular, en el sentido ya indicado, determinadas reglas deductivas" ${ }^{15}$

Lo que en el campo de la lógica clásica vale para los conceptos, los juicios y los raciocinios en general, es aplicable, mutatis mutandis, a los conceptos, los juicios y los raciocinios jurídicos.

Por ejemplo: la fórmula estenográfica: 'Si $s$ es $h, O$ debe asumir la conducta $d$ y $P$ puede lícitamente observar el comportamiento $f$, exhibe la estructura de la regulación jurídica o, en otras palabras, indica qué elementos de la misma son constantes y cuáles no tienen tal carácter. ${ }^{16}$ Conocemos así la forma de aquélla y podemos distinguir - relativamente a las normas prescrip-

13 Scholz, op. cit., \$1', pág. 3.

14 Scholz, op. cit., $\$ 1$, pág. 3.

15 Scholz, op. cit., \$1, pág. 4.

16 Eduardo García Máynez, Lógica del Juicio Jurídico, Cap. II. 
tiva y atributiva - los elementos variables e invariables de ambos juicios. En el cuso del ejemplo trátase de una 'forma lógica perfecta', porque si sustituimos adecuadamente los signos de las variables, obtenemos un juicio del que es dable predicar — dado su carácter normativo- ya la validez, ya la invalidez.

Para distinguir dos o más conceptos, dos o más juicios, o dos o más raciocinios jurídicos es necesario tener en cuenta su contenido o, en otras palabras, el elemento variable de aquéllos. Por ejemplo: la forma lógica de las nociones compuestas 'derecho de ejercicio potestativo' y 'derecho de ejercicio obligatorio’ es idéntica, y lo único que permite diferenciarlas es su aspecto material. Análogamente, los silogismos jurídicos que siguen poseen los mismos elementos formales, y sólo difieren en el otro aspecto:

1) 'El que sin mandato y sin estar obligado a ello se encarga de un asunto de otro, debe obrar conforme a los intereses del dueño del negocio;

'Primus se ha encargado de tal o cual asunto de Secundus, sin mandato de éste y sin estar obligado a ello; luego:

'Primus debe obrar, en el manejo de ese asunto, conforme a los intereses de Secundus'.

2) El que sin causa se enriquece en detrimento de otro, está obligado a indemnizarlo de su empobrecimiento en la medida en que se ha enriquecido';

' $X$ se ha enriquecido sin causa en detrimento de $Y$ '; luego:

' $X$ debe indemnizar a $Y$ de su empobrecimiento, en la medida en que se ha enriquecido'.

En todos estos casos trátase de estructuras lógicas o, para hablar con mayor rigor, de formas de objetos lógicos. Podemos sostener, por tanto, que las discursivas de que ahora tratamos corresponden siempre a conceptos, a juicios o a raciocinios, y exhiben lo que de invariable o constante hay en esas significaciones.

5. En la esfera jurídica no sólo puede hablarse - ni sólo se ha habladode formas lógicas o, en otros términos, de formas de conceptos, de raciocinios o de juicios. En ella existen igualmente formas de otra especie, que no se refieren ya a los conceptos, a los juicios o a los raciocinios, sino a sus correlatos objetivos. No sb́lo en los objetos lógico-jurídicos, también en los ontológicojurídicos encontramos elementos de orden formal y material. La "geometría del fenómeno. jurídico" - a que alude Kelsen en una página de sus Hauptprobleme- es, en realidad, una teoría sobre los elementos formales de los objetos jurídicos, ya se trate de los insertos en el plano lógico, ya de los que pertenecen al ontologico. ${ }^{17}$ Estamos, pues, dada la naturaleza de aquellos objetos, en

17 Hans Kelsen, Hauptprobleme der Staatsrechtslehre, Verlag von J. C. B. Mohr, Tübingen, 1911; pág. 93. Sobre la distinción entre plano gramatical de las oraciones, plano lógico de los juicios normativos y plano ontologico de las situaciones a que éstos se refieren, véase lo expuesto en mi Lógica del Juicio Jurídico, Cap. I, 1. 
presencia de una especie de geometría de formas inextensas, referida a objetos ideales. Que un argumento analógico o un derecho de crédito - para poner ejemplos de objetos que pertenecen a los dos planos- se hallan insertos en el orden de la idealidad - y no en el del ser real- resulta evidente, pues nadie ha 'visto' o 'tocado', ni puede aprehender a través de sus sentidos, ninguna de esas entidades. $Y$ aun cuando ambas pertenezcan al ser ideal, entre ellas existe una diferencia, pues mientras la forma de la primera es lógica o discursiva, la de la segunda no tiene tal carácter, ya que los derechos de crédito -como en general los subjetivos- no son conceptos, ni juicios, ni raciocinios.

¿En qué sentido cabe afirmar que un derecho subjetivo o un deber jurídico, por ejemplo, poseen un contenido y una forma?

Si frente a dos o más facultades legales indagamos en qué coinciden y en qué difieren, sin esfuerzo podremos percatarnos de que lo que tienen en común es precisamente ser derechos subjetivos, mientras que su diversidad depende del contenido u objeto de cada una.

¿Por qué decimos que se trata de derechos subjetivos? $O$, en otros términos: ¿por qué sostenemos que son objetos del mismo género? La respuesta a tales interrogantes $y$, en general, a la pregunta sobre lo que determina la pertenencia de un objeto a una clase, es muy sencilla: lo que condiciona tal pertenencia es la posesión - por parte de algo- de los atributos que todos los miembros de la clase tienen en común. Se trata - para decirlo de otro modo- de las condiciones que en forma necesaria $y$ suficiente determinan la inclusión de cada uno en la misma clase. Y como - para retomar el ejemplo- la definición del derecho subjetivo indica qué tienen en común todos los miembros de la clase designada por el correspondiente concepto, a fin de cuentas resulta que los elementos invariables de esos objetos son, precisamente, los definitorios. Y Y así como en el caso de los conceptos, los raciocinios y los juicios dimos el calificativo de formales a los elementos constantes de éstos, en el de los ontológico-jurídicos nada impide sostener que su forma está condicionada por los elementos invariables -0 , lo que es igual, por la definición- de tales objetos.

En otra obra hemos definido el derecho subjetivo como la posibilidad, concedida a una persona por una norma, de hacer o de omitir licitamente algo. ${ }^{18}$ Usando giros aristotélicos podría decirse que el género próximo queda indicado por las palabras 'posibilidad de hacer o de omitir algo'. Los derechos subjetivos no son hechos, sino meras posibilidades de acción o de omisión. El dueño de una finca tiene la posibilidad de vivir o no vivir en ella; la de rentarla, venderla, permutarla, etc.; pero ninguna de estas posibilidades es un hecho. La realización de cualquiera de ellas, en cambio, sí tiene tal carácter,

18 Eduardo García Máynez, Introducción a la Lógica Juridica, Fondo de Cultura Económica, México, 1951; Cap. VI, 22, pág. 230. 
por ser una forma de conducta; $y$, precisamente porque estamos ante la realización de una de esas posibilidades en que el derecho consiste, declaramos que el comportamiento que la realiza implica el ejercicio de la facultad legal. No se trata, simplemente, de la realización de una conducta posible - por parte del sujeto facultado - sino de un acto (o de una omisión) que la norma que atribuye el derecho considera como formas de ejercicio del mismo. La posibilidad que al realizarse puede ser vista como acto de ejercicio, no es una posibilidad cualquiera (una mera posibilidad de hecho), sino normativamente calificada: la de hacer u omitir licitamente algo. El adverbio lícitamente' indica - para valernos otra vez de giros aristotélicos- la diferencia específica del concepto, y revela que sólo pertenecen a la clase de los derechos subjetivos las posibilidades de conducta que al realizarse ostentan el signo positivo de la licitud. Y como sostener que un comportamiento es lícito equivale a decir que está jurídicamente permitido, el derecho subjetivo puede igualmente definirse como permisión normativa de una conducta (acción $u$ omision). Declarar que un sujeto tiene el derecho de hacer (o el de omitir) algo, y decir que una norma jurídica le permite hacerlo (u omitirlo), es enteramente lo mismo.

Podemos, pues, sostener que en el derecho subjetivo la forma consiste en la permisión de un comportamiento, y el contenido en lo que en cada caso el titular puede lícitamente hacer o no hacer. Lo que distingue a dos o más facultades jurídicas es precisamente el susodicho elemento material; la forma, en cambio, es idéntica en todas, pues todas consisten en una permisión. Sólo cuando sabemos qué es lo que un sujeto puede lícitamente hacer u omitir, estamos en condiciones de indicar concretamente el objeto o contenido del derecho, y de establecer diferencias entre éste y cualquiera otro del mismo género, ya de la misma persona, ya de un sujeto diverso. La palabra 'algo', en la primera de las dos definiciones, desempeña el mismo papel que en las fórmulas lógico-matemáticas corresponde al signo de la variable. Si bien el contenido es elemento esencial de todo derecho, tal elemento es -por esencia-variable. Mejor dicho: variable es el objeto específico de cada facultad (lo en cada caso permitido o facultado); invariable, en cambio, la presencia de ese elemento que permite distinguir un derecho de otro, y que, unido a los demás definitorios, exhibe la forma de todos los miembros de la clase. Ello revela que el contenido es el elemento individualizador de los derechos subjetivos $\mathrm{y}$, en general, de todo objeto del conocimiento jurídico.

Si comparamos nuestra obligación de pagar determinado impuesto, con la de devolver un libro que nos fue prestado, inmediatamente advertiremos que el contenido de esos deberes es el elemento distintivo de ambos. La conducta objeto del deber es en un caso el pago de cierta suma; en el otro, la devolución de un libro. $\mathrm{Y}$ aun cuando los dos deberes tienen su propio objeto (al lado de otros elementos formales), el contenido de aquéllos varía 
en cada caso. La forma, empero, es la misma, pues en ambos se trata de deberes jurídicos. Y como el deber jurídico es la restricción de la libertad exterior de una persona, derivada de la facultad, concedida a otra $u$ otras, de exigir de la primera una acción o una omisión, los elementos formales de todo deber son los que la anterior definición señala.

El deber jurídico restringe la libertad de acción del obligado, en cuanto le niega el derecho de omitir la conducta prescrita o el de ejecutar la prohibida, y sólo le concede el de hacer lo que se le manda o el de omitir lo que se le veda. Tal restricción deriva siempre del derecho, correlativamente concedido a otro (u otros), de exigir del obligado que haga (u omita) algo. Aquí, también, la palabra 'algo' apunta al 'objeto' del deber, es decir, al elemento variable del mismo, aun cuando uno de los constantes o esenciales sea la presencia del que en cada caso cambia.

Así como la forma 'atributiva' de regulación del comportamiento ( 0 , en otras palabras, la que concede el derecho subjetivo) consiste siempre en la permisión de algo (lo que supone que hay una norma que permite, un sujeto a quien se permite, una permisión y una conducta permitida), la forma 'prescriptiva' (esto es, la que impone el deber jurídico), en todo caso implica una prohibición: ora la de omitir la conducta ordenada, ora la de ejecutar la prohibida. Lo cual revela, al propio tiempo, que el destinatario de las disposiciones que ordenan o prohiben -el obligado-nunca es libre (en el sentido jurídico del término) relativamente al objeto o contenido de la prohibición o del mandato, ya que no puede lícitamente optar entre la ejecución y la omisión de la conducta prohibida u ordenada. ${ }^{19}$

Como a cada concepto lógico-jurídico corresponde otro ontológico-jurídico y, además, todo concepto tiene un correlato, resulta obvio que el número de 'formas' y 'contenidos' es el mismo en cada uno de los correspondientes planos. Y esto vale no sólo para los conceptos, sino para los demás objetos lógicos, ya que todos tienen un correlato en el plano ontológico. A toda norma de derecho, por ejemplo, corresponde en ese plano una relación jurídica, y ésta, como los demás objetos de su clase, posee también un contenido y una forma. ${ }^{20}$

La única diferencia, relativamente a los insertos en cada uno de esos planos, consiste, como hemos visto, en que la 'forma' de los lógico-jurídicos (conceptos, juicios y raciocinios), es discursiva, en tanto que la de los otros no ostenta ese carácter. Pero, en ambos casos, el elemento constante es el formal, y lo que varía es el contenido.

La distinción entre elementos materiales y formales aplícase tanto a los conceptos implícitos en la noción universal del derecho cuanto a sus correlatos objetivos, lo mismo que a los conceptos de menor generalidad subordinados a los primeros y a los correlatos de esos conceptos menos generales. En otras

19 Introducción a la Lógica Jurídica, Cap. VI, 13, pág. 206.

20 Cf. Eduardo García Máynez, Lógica del Juicio Jurídico, Cap. I, 2, y Cap. VI, 9. 
palabras: todo objeto del conocimiento jurídico -ya en el plano lógico, ya en el ontológico- posee un contenido y una forma, sea cual fuere la amplitud de la noción correspondiente. Lo dicho puede, por ejemplo, aplicarse lo mismo al correlato objetivo del término 'deber jurídico', que al del concepto 'deber jurídico del comprador de una cosa’. Lo propio cabe afirmar -para poner otro ejemplo- del derecho subjetivo (genéricamente considerado) y un derecho real o un derecho de crédito.

Para definir los conceptos jurídicos que Somló llama 'fundamentales' no basta, pues, el conocimiento de la distinción que hemos venido estudiando, ya que ésta es aplicable a todos los objetos jurídicos, sea cual fuere el plano en que estén insertos o la mayor o menor generalidad de las correspondientes nociones. Lo único que hemos puesto en claro es que la Teoría Jurídica Fundamental no se interesa por los contenidos, sino por la forma de ciertos objetos: en primer término, por la del derecho en general y su concepto; en segundo lugar, por la de las nociones fundamentales implícitas en tal concepto y la de los correlatos objetivos de aquéllas.

De esta suerte introducimos una primera modificación en la tesis del jurista húngaro, $\mathrm{y}$ ampliamos su alcance, al distinguir los conceptos jurídicos fundamentales y sus correlatos objetivos. No es lo mismo, en efecto, la noción universal del derecho que el objeto a que está referida, ni podemos tampoco confundir el derecho subjetivo o el deber jurídico, como tales, con el concepto del derecho subjetivo o el del deber jurídico. La Teoría Jurídica Fundamental no sólo debe inquirir la 'forma' de los conceptos fundamentales, sino la de sus correlatos objetivos, sea cual fuere el plano en que aparezcan. No se trata, pues, solamente de estudiar la 'forma' de esos conceptos, sino la de todos los objetos logico-jurídicos (juicios, por ejemplo), de carácter fundamental y, naturalmente también, la de los correlatos objetivos correspondientes. En otras palabras: la teoría de la 'formas' jurídicas fundamentales comprende dos grandes ramas: en cuanto estudia 'formas discursivas' es Lógica Jurídica; en cuanto se refiere a 'formas no discursivas' se llama Ontología Formal del Derecho. La última puede definirse como estudio de las 'formas' de los objetos ontológico-jurídicos que fungen como correlatos de los lógico-jurídicos fundamentales. La relación entre Ontología Formal del Derecho y Lógica Jurídica es muy íntima, ya que -como lo hemos demostrado en anteriores trabajos- la primera de las dos constituye la base o el fundamento de la otra. ${ }^{21}$

Nuestro problema inmediato estriba, por tanto, en saber qué conceptos jurídicos merecen el calificativo de fundamentales. $\mathrm{Y}$ es obvio que la distinción entre elementos formales y materiales tendrá que aplicarse no solamente a esos conceptos, sino a sus correlatos, entre los que hay objetos lógicos que no son conceptos, como - para poner dos ejemplos- los llamados norma

21 Cf. Introducción a la Lógica Jurídica, Cap. V. 
jurídica atributiva y norma jurídica prescriptiva, que fungen como elementos invariables de la regulación bilateral del comportamiento humano.

6. En nuestra opinión, el método adecuado para determinar qué nociones jurídicas son fundamentales, es el que proponen Stammler y Somló, el primero en su Theorie der Rechtswissenschaft (1911) y el segundo en su Juristische Grundlehre (1917, segunda edición, 1926).

Tales conceptos - escribe Rodolfo Stammler - son "irradiaciones formales" de la noción universal del derecho, o "funciones lógicas" de toda consideración jurídica, y deben ser derivados del análisis de esa noción básica. "La prueba de que una cierta dirección formal del pensamiento pertenece a la clase de los conceptos jurídicos fundamentales, y la de que el número de éstos es limitado y fijo, se obtienen por aplicación del método que consiste en llegar hasta los componentes elementales en cuya síntesis unitaria consiste el concepto mismo del derecho." 22

Si las conclusiones a que llegan en este punto los dos famosos juristas no son admitidas por nosotros, ello simplemente obedece a que no aceptamos su punto de partida, esto es, sus definiciones del derecho. El método que proponen nos parece correcto; mas no podemos pensar lo mismo ni de los supuestos generales de sus respectivas doctrinas, ni, especialmente, de las definiciones que utilizan para la derivación de los conceptos jurídicos puros.

7. Veamos ahora qué conclusiones podemos obtener, tomando como base la definición formulada por nosotros en anteriores trabajos. ${ }^{23}$ Si lo que distingue al derecho de otras formas de regulación normativa es la nota de bilateralidad, el cuadro de las nociones jurídicas básicas tendrá que poder derivarse del concepto de regulación bilateral. ${ }^{24}$

8. Examinemos qué conceptos lógico-jurídicos están implícitos en la definición de esta última. Después habrá que inquirir cuáles corresponden a los objetos ontológico-jurídicos a que aquellos conceptos se refieren.

Si la regulación bilateral está constituida por la conexión necesaria y recíproca de una norma que faculta y otra que impone deberes, los conceptos de norma jurídica atributiva y norma jurídica prescriptiva tendrán que ser clasificados como fundamentales, ya que están implícitos en el de regulación bilateral. Y pertenecen al plano lógico-jurídico porque son conceptos de objetos-lógicos o, más concretamente, porque están referidos a juicios.

Para obtener la tabla completa de los conceptos lógico-jurídicos fundamentales hay, pues, que hacer un análisis de los elementos de los juicios atri-

22 R. Stammler, op. cit., pág. 115.

23 Cf. El problema de la definición del derecho y Lógica del Juicio Jurídico, Cap. II.

24 Lógica del Juicio Jurídico, Caps. I y II. 
butivo y prescriptivo o, lo que es igual, decir qué conceptos los componen.

Como en ambos casos se trata de normas hipotéticas que hacen depender de la realización de sus supuestos determinadas consecuencias de derecho, obvio es que entre los conceptos lógico-jurídicos fundamentales deben figurar los correspondientes a las dos partes de esas normas, esto es, los de supuesto jurídico y disposición normativa. Que están implícitos en los anteriores es evidente, puesto que, por definición, las normas jurídicas atributiva y prescriptiva tienen el carácter de juicios en que el nacimiento del derecho y del deber se encuentra condicionado por la realización de una hipótesis. Los correlatos de aquellos conceptos son de orden lógico, porque se trata de partes de juicios jurídicos $\mathrm{y}$, por tanto, de significaciones.

Si ahora recordamos cuáles son los elementos de la disposición de cada uno de esos juicios, nuestra lista de las nociones lógico-jurídicas fundamentales quedará completa. Cada una de las dos disposiciones normativas consta de tres elementos: conoepto-sujeto, cópula jurídica y predicado relacional. Podemos, pues, por un lado, referirnos al concepto-sujeto de la norma atributiva, a la cópula atributiva y al predicado de la norma que faculta y, por otro, al concepto-sujeto de la norma que prescribe, a la cópula prescriptiva y al predicado relacional de la norma prescriptiva. ${ }^{25}$ Todos son conceptos de conceptos, puesto que hacen referencia a los elementos constitutivos de las dos disposiciones.

De acuerdo con lo dicho, el cuadro de los conceptos lógico-jurídicos fundamentales es éste:
I) Norma atributiva
Supuesto jurídico de la norma atributiva Disposición atributiva
$\{$ Concepto-sujeto de la norma atributiva Cópula jurídica atributiva Predicado relacional de la norma atributiva
II) Nonma prescriptiva $\left\{\begin{array}{l}\text { Supuesto jurídico de la norma prescriptiva. } \\ \text { Disposición prescriptiva } \\ \text { Concepto-sujeto de la norma prescriptiva. } \\ \text { C6pula jurídica prescriptiva } \\ \text { Predicado relacional de la norma prescriptiva }\end{array}\right.$

De estos conceptos, los de supuesto jurídico de la norma atributiva y supuesto jurídico de la prescriptiva difieren sólo formalmente (en cuanto elementos de dos normas distintas); pero coinciden materialmente, puesto que la hipótesis de ambas es igual.

El análisis completo de tales normas revela, pues, que los conceptos

25 Eduardo García Máynez, “Clasificación de los conceptos jurídicos", en Diánoia, Anuario del Centro de Estudios Filosóficos, 1956. 
lógico-jurídicos fundamentales son 12. Es claro que si no llevamos el análisis hasta este extremo y, en vez de hablar, por ejemplo, de norma jurídica atributiva y norma jurídica prescriptiva, nos referimos solamente al concepto de norma jurídica, que abarca a los otros otros, el número de los conceptos lógicojurídicos fundamentales, en este grado de mayor generalidad, quedará reducido a la mitad, como lo indica el cuadro que sigue:

$$
\text { Norma jurídica }\left\{\begin{array}{l}
\text { Supuesto jurídico } \\
\text { Disposición normativa } \\
\text { Concepto-sujeto } \\
\text { Cópula jurídica } \\
\text { Predicado relacional }
\end{array}\right.
$$

9. Para trazar el cuadro de los conceptos ontológico-jurídicos fundamentales habrá que añadir, a los anteriores lógico-jurídicos, los correspondientes a los objetos que fungen como sus correlatos en el otro plano. Por ejemplo: al concepto disposición de la norma atributiva corresponde, en el plano ontológico, el de relación jurídica directa. Este concepto es, pues, ontológicojurídico, ya que no se refiere a una parte de la norma atributiva, sino al vínculo creado por tal norma entre el sujeto del derecho y el sujeto del deber.

Como a cada uno de los objetos insertos en el plano lógico-jurídico corresponde otro en el otro plano, el número de los conceptos correspondientes a los dos es siempre el mismo. Así lo indica el cuadro que sigue:

\section{Conceptos: lógico-jurídicos}

节

1. Supuesto de la norma atributiva

2. Disposición atributiva

3. Concepto-sujeto de la norma atributiva

4. Cópula atributiva

5. Predicado de la norma atributiva

1. Supuesto de la norma prescriptiva

2. Disposición prescriptiva

3. Concepto-sujeto de la norma prescriptiva

4. Cópula prescriptiva

5. Predicado de la norma prescriptiva
Conceptos ontológico-jurídicos

1. Hecho condicionante de la relación jurídica directa

2. Relación jurídica directa

3. Pretensor

4. Derecho subjetivo

5. Objeto del derecho

1. Hecho condicionante de la relación jurídica conversa

2. Relación jurídica conversa

3. Obligado

4. Deber jurídico

5. Objeto del deber 